\title{
Automated EEG discontinuity in cooled newborns predicts cerebral MRI and neurodevelopmental outcome
}

Jonathan M Dunne ${ }^{1}$, David Wertheim MA, $\mathrm{PhD}^{2}$; Paul Clarke MD, FRCPCH ${ }^{3}$; Olga Kapellou MRCPCH, MD, PhD ${ }^{4}$; Philippa Chisholm MRCPCH ${ }^{4}$; James P Boardman PhD, FRCPCH ${ }^{5}$ and Divyen K Shah MBChB, PhD ${ }^{1,6}$

${ }^{1}$ Barts and the London Medical School, London, UK

${ }^{2}$ Kingston University, London, UK

${ }^{3}$ Norfolk and Norwich University Hospitals NHS Foundation Trust, Norwich, UK

${ }^{4}$ Homerton University Hospital, London, UK

${ }^{5}$ MRC Centre for Reproductive Health, University of Edinburgh, UK

${ }^{6}$ Royal London Hospital, London, UK

Corresponding Author: Dr Divyen K Shah, Royal London Hospital, London E1 1BB.

T: +44 2035940524 E: divyen.shah@bartshealth.nhs.uk

Short title: EEG discontinuity in cooled newborns

Key words: EEG, discontinuity, MRI, neurodevelopment, therapeutic hypothermia

Abbreviations: $\mathrm{TH}$ - therapeutic hypothermia

Financial disclosure statement: The authors have no financial relationships relevant to this article to disclose.

Funding source: JPB received funding support from Theirworld

Potential conflicts of interest: None

\section{What is known about this topic:}

EEG discontinuity is associated with adverse neurologic outcomes in term infants after hypoxic-ischaemic encephalopathy.

\section{What this study adds:}

In term infants receiving therapeutic hypothermia, EEG discontinuity calculated using a novel algorithm at 24 hours is associated with cerebral tissue injury on MRI and with adverse neurodevelopmental outcomes. Therefore, it may provide a useful tool for early risk stratification when adjunctive therapies are most beneficial. 


\section{Contributors' Statement:}

Jonathan M Dunne collected data, carried out the raw EEG data analysis, assisted with the statistical analysis, wrote the first draft of the manuscript and approved the final version of the manuscript.

David Wertheim developed the EEG software used to measure discontinuity, assisted with writing the manuscript and approved of the final version of the manuscript.

Paul Clarke, obtained ethics approval, collected data, assisted with writing the manuscript and approved the final version of the manuscript.

Olga Kapellou reviewed the MR images and approved of the final version of the manuscript.

Philippa Chisholm carried out neurodevelopmental testing and approved the final version of the manuscript.

James P Boardman reviewed the MR images, assisted with writing the first draft of the manuscript, assisted with statistical analysis and approved the final version of the manuscript.

Divyen K Shah conceived the study, collected data, assisted with the EEG reviews, statistical analysis, writing the first draft of the manuscript, approved of the final version of the manuscript and is guarantor.

Word Count: 2498 


\begin{abstract}
Background and Hypothesis: Prolonged EEG discontinuity has been associated with poor neurodevelopmental outcomes after perinatal asphyxia but its predictive value in the era of therapeutic hypothermia $(\mathrm{TH})$ is unknown. Hypothesis: In infants undergoing TH for hypoxicischaemic encephalopathy (HIE) prolonged EEG discontinuity is associated with cerebral tissue injury on MRI and adverse neurodevelopmental outcome.
\end{abstract}

Method: Retrospective study of term neonates from 3 UK centres who received TH for perinatal asphyxia, had continuous 2 channel aEEG with EEG for a minimum of 48 hours, brain MRI within 6 weeks of birth, and neurodevelopmental outcome data at a median age of 24 months. Mean discontinuity was calculated utilising a novel automated algorithm designed for analysis of the raw EEG signal.

Results: Of 49 eligible infants, 17 (35\%) had MR images predictive of death or severe neurodisability (unfavourable outcome) and 29 (59\%) infants had electrographic seizures. In multivariable logistic regression, mean discontinuity at 24 hours and 48 hours (both $p=0.01$ ), and high seizure burden $(\mathrm{p}=0.05)$ were associated with severe cerebral tissue injury on MRI. A mean discontinuity $>30$ s per minute-long epoch, had a specificity and positive predictive value of $100 \%$, sensitivity of $71 \%$ and a negative predictive value of $88 \%$ for unfavourable neurodevelopmental outcome at a $10 \mu \mathrm{V}$ threshold.

Conclusions: In addition to seizure burden, excessive EEG discontinuity is associated with increased cerebral tissue injury on MRI and is predictive of abnormal neurodevelopmental outcome in infants treated with TH. The high positive predictive value of EEG discontinuity at 24 hours may be valuable in selecting newborns with HIE for adjunctive treatments. 


\section{Introduction}

Hypoxic-ischaemic encephalopathy (HIE) affects up to 6 per 1000 live births in industrialised nations and is an important cause of disability in survivors ${ }^{12}$. Mild therapeutic hypothermia (TH) is a safe and effective neuroprotective intervention and is now standard care in resourcerich settings ${ }^{34}$. TH increases the number of survivors without neurodisability, with the benefits persisting into childhood ${ }^{5}$. The number needed to treat is nine ${ }^{4}$, so there is a need to stratify patients who may stand to benefit from adjunctive therapies, and to improve the precision of prognostic information for families and clinicians.

In the precooling era, prolonged EEG discontinuity of 30s or longer in term newborns with $\mathrm{HIE}^{6}$, as well as in other conditions including cerebral haemorrhage and infection ${ }^{7}$, was associated with unfavourable neurodevelopmental outcomes. However, the applicability of EEG quantification for infants undergoing TH after HIE is unclear. Continuous limited channel EEG with amplitude-integrated EEG (aEEG) monitoring is routinely used at the bedside in newborns undergoing TH for assessment of electrocortical background and to screen for seizures $^{8}$. We have previously shown that electrographic seizure burden is independently associated with cerebral tissue injury on MRI in term-born infants undergoing $\mathrm{TH}^{8}$.

Severely abnormal EEG and aEEG background patterns are associated with adverse outcome ${ }^{9}$ ${ }^{10}$, but the precise features of the background that predict poor outcome are uncertain and qualitative assessments lack diagnostic precision and are susceptible to inter-observer variation. In order to improve the objectivity of EEG assessment in the newborn, we developed and applied computer software for automated quantification of EEG discontinuity.

We aimed to test the hypothesis that an objective measure of EEG discontinuity is associated with cerebral tissue injury on MRI and neurodevelopmental outcome. 


\section{Method}

We studied infants who received TH between October 2007 and July 2011 in three tertiary UK Neonatal Intensive Care Units (NICUs): the Royal London Hospital, Homerton University Hospital, and Norfolk and Norwich University Hospital. Data were obtained as part of standard clinical care. This study had Research Ethics Committee approval (UK REC reference 14/EE/0205).

\section{Subjects}

Neonates were eligible for inclusion if they were born at $\geq 36$ weeks' gestation, treated with whole body TH for moderate to severe HIE (defined using standard criteria) ${ }^{11}$, had aEEG monitoring within 12 hours of birth, and had brain MRI within 6 weeks. Exclusion criteria included death prior to MR imaging, images degraded by motion artefact, major congenital anomaly or a primary diagnosis of an inborn error of metabolism. The cohort is a subset of a larger cohort reported previously ${ }^{8}$.

Infants received intensive care and $\mathrm{TH}$ according to local guidelines, which were informed by the UK TOBY Cooling Register Clinician's Handbook ${ }^{12}$. Sentinel events were defined as a sustained (pre-terminal) fetal bradycardia necessitating delivery of the infant, antepartum haemorrhage secondary to placental abruption, placenta praevia, cord prolapse or rupture, uterine rupture and shoulder dystocia.

\section{aEEG/EEG monitoring and discontinuity analysis}

Recordings obtained from the aEEG monitors (BRM2/3 BrainZ Instruments, Natus Medical Inc., CA, USA) were reviewed off-line (by DS and JD) to visually identify seizure-free and minimum artefact 2-hour epochs at 24 and 48 hours of age. Single channel cross-cerebral (P3P4) EEG data were exported to Excel, and continuity was analysed in 1-minute epochs using software that we developed using MATLAB (The MathWorks, Inc., MA, USA), similar to that validated in previous work by comparing with visual assessment ${ }^{13}$. The system detected an 
interval if the absolute amplitude of the EEG was less than $15 \mu \mathrm{V}$ with respect to the baseline for at least 6 seconds and therefore discontinuous; the analysis was repeated with a $10 \mu \mathrm{V}$ threshold. The 10 and $15 \mu \mathrm{V}$ thresholds were chosen to reflect the fact that the EEG from healthy term newborns is represented by more lower voltage high frequency wave forms in contrast to preterm or encephalopathic infants ${ }^{14}$. For each recording the mean of the total interval length per epoch, the discontinuity value, was calculated and expressed in seconds. For an interval to be detected the minimum period of 6 seconds was chosen in order to avoid including normal short quiescent periods seen in tracé alternant ${ }^{15}$. In order to allow assessment of temporal variability in EEG discontinuity, the length of the analysis epochs was one minute; intervals that started in the preceding epoch and continued into a subsequent period were included in the analysis. EEG discontinuity greater than $30 \mathrm{~s}$ per minute was regarded as a predominantly discontinuous trace, as this equates to $50 \%$ discontinuity within a minute implying that the EEG is predominantly discontinuous at the given amplitude threshold. This is similar to approaches previously applied in visual assessment of term infant EEG recordings ${ }^{7}$. For four traces, the analysis was repeated which yielded the same mean interval duration.

\section{MR Imaging}

Magnetic resonance imaging was performed at local centres with conventional T1-weighted and T2-weighted sequences acquired at 1.5 Tesla. Anonymised images were rated independently by two investigators (OK and JPB), who were blind to the clinical data. The pattern of MRI injury was classified into two groups, using the system described by Rutherford et al. ${ }^{16}$, which has prognostic value in the era of TH. This method includes rating the posterior limb of the internal capsule (PLIC), basal ganglia and thalami (BGT) and the subcortical white matter. Group 1 had a severe pattern of injury that predicts poor outcome, defined by the classification system as death or one or more of: mental development index (MDI) score less than 70 ( $\geq 2$ SD below the mean) on the Bayley Scales of Infant Development (BSID II); score of 3-5 on the gross motor function classification system (GMFCS); or bilateral cortical visual 
impairment with no useful vision. Group 2 had either normal images or less severe patterns of injury associated with normal or mildly abnormal (favourable) neurodevelopmental outcome. These patterns of injury were used to assign infants into either the favourable or unfavourable MR images outcome group.

\section{Neurodevelopmental Outcomes}

For the purposes of neurodevelopmental outcomes, infants were classified in the unfavourable outcome group if they had cerebral palsy that impaired independent walking; severe seizure disorder; hearing impairment requiring hearing aids or bilateral cortical visual impairment with no useful vision. Infants with better outcomes were placed in the favourable outcome group. Neurodevelopmental outcomes were assessed at multiple local centres, with tools of assessment including both BSID and Griffiths.

\section{Statistical Analysis}

Data were analysed using SPSS V22 (IBM). We investigated discontinuity at 10 and $15 \mu \mathrm{V}$ thresholds at both 24 and 48 hour time epochs, seizure burden, Apgar score at 10 minutes, use of anticonvulsants, worst arterial cord or infant $\mathrm{pH}$ and base deficit within the first hour in univariable analyses with cerebral tissues injury on MRI using $\chi^{2}$ statistic for categorical variables and analysis of variance (ANOVA) for continuous variables. Seizures were classified as previously reported for this cohort ${ }^{8}$. Seizures were defined as rhythmic spike and/or wave activity on the raw EEG lasting at least $10 \mathrm{~s}$ in the absence of artefact. A low seizure burden was defined as no seizures or sporadic seizures lasting $<15$ minutes in a single hour. A seizure episode lasting $\geq 15$ minutes per hour for any hour during the period of monitoring including the cooling and rewarming period was classified as high seizure burden.

Significant factors from the univariable analyses were examined in a binary logistic regression model to calculate odds ratios (OR), assessing their association with MRI outcome. The area under the receiver operating characteristic (ROC) was calculated for the relationship between 
discontinuity $>30$ s and unfavourable MRI outcomes. The OR, positive predictive value (PPV), negative predictive value (NPV), sensitivity and specificity were calculated for the relationship between discontinuity $>30$ s and unfavourable neurodevelopmental outcomes. Two-sided $p$ values were regarded as significant at the 0.05 threshold. 


\section{Results}

Of 52 eligible infants, three were excluded due to incomplete EEG recordings at 48 hours. The clinical characteristics of the remaining 49 are described in Table 1 and the EEG discontinuity measures, MRI findings and neurodevelopmental outcomes are detailed in the Table 2.

\section{MRI Outcomes}

Seventeen $(35 \%)$ of the study cohort had MR images predictive of unfavourable outcome.

On univariable analysis, factors associated with the unfavourable pattern of MRI outcome were high seizure burden $(\mathrm{p}=0.003)$, discontinuity at 24 hours $(\mathrm{p}<0.001)$ and at 48 hours $(\mathrm{p}<0.001)$ at $15 \mu \mathrm{V}$ threshold (Table 3).

In multivariable logistic regression high seizure burden (OR 4.2, 95\% CI 1.01-17.48; $\mathrm{p}=0.05$ ), mean discontinuity at 24 hours (OR 1.04, 95\% CI 1.01-1.08; $\mathrm{p}=0.01)$ and at 48 hours (OR 1.05, 95\% CI 1.01-1.10; $\mathrm{p}=0.01$ ) were associated with severe cerebral tissue injury on MRI (using the method described by Rutherford et $\mathrm{al}^{16}$ ). Of infants with unfavourable MRI outcomes, the median (IQR) discontinuity in seconds was $31.2(0.9,51.8)$ and $43.5(8.6,56.5)$ at 10 and 15 $\mu \mathrm{V}$ respectively at 24 hours, and $2.7(0.1,42.7)$ and $20.3(1.9,53.2)$ at 48 hours (Figure 1$)$. A mean discontinuity $>30$ s per 1 -minute epoch had a PPV of $90 \%$ and $86 \%$ at 24 and 48 hours respectively (10 $\mu \mathrm{V}$ threshold) and of $75 \%$ and $80 \%$ at 24 and 48 hours (15 $\mu \mathrm{V}$ threshold) for group 1 (unfavourable MRI outcome), The area under the ROC curve at both thresholds and both time points was between 0.78 and 0.81 showing a high predictive value for discontinuity at both time points and thresholds. To investigate the possibility of collinearity between seizure burden and discontinuity ( 24 hours, $10 \mu \mathrm{V}$ threshold) in the regression model we calculated correlation between these two variables and found the Pearson coefficient to be low, 0.30 $(\mathrm{p}=0.036)$ 


\section{Neurodevelopmental Outcomes}

Neurodevelopmental outcomes were available for $43 / 49(88 \%)$ of the children (Table 2). Median age (interquartile range) of last follow-up was $24(24,24)$ months. 14/43 (33\%) children had unfavourable clinical outcomes: four had died, six had cerebral palsy, two required bilateral hearing aids for profound sensori-neural hearing loss, one had autism, seizures and global speech delay and one had seizures and unilateral hearing loss (Table 2).

Ten of the 14 children with unfavourable outcomes had a discontinuous trace (>30s). For these 14 children, the median (range) discontinuity in seconds was $50.3(9.6,55.1)$ and $55.2(24.8$, 58.0) at 10 and $15 \mu \mathrm{V}$ respectively at 24 hours and $18.1(0.1,47.7)$ and $42.8(5.3,53.3)$ at 48 hours (Figure 2). A mean discontinuity $>30$ s per 1 -minute epoch has a PPV of $100 \%$ and $87 \%$ at 24 and 48 hours respectively $(10 \mu \mathrm{V}$ threshold) and of $91 \%$ and $90 \%$ at 24 and 48 hours (15 $\mu \mathrm{V}$ threshold) for unfavourable neurodevelopmental outcome (Table 3).

Of the 14 children with unfavourable clinical outcomes, 10 had MR images predictive of unfavourable outcome. Of the remaining four children, two had profound bilateral hearing loss and one had seizures and unilateral hearing loss, which the MRI system does not predict. One infant died at 6 months from complications of evolving cerebral palsy.

For the 29 children with favourable neurodevelopmental outcomes only one had EEG discontinuity $>30$ s at both time epochs and voltage thresholds. Six of these 29 had MRIs that would predict likely neurodisability: three of these children had neurodevelopmental abnormalities including mildly impaired cognition, and/or delayed speech and language development, and three were classified as normal at the time of assessment. Overall, the correlation between MRI and neurodevelopmental outcome was good: Pearson's R=0.62 $(\mathrm{p}<0.001)$. Of the six children lost to follow-up, five had MRI predictive of favourable outcomes. 


\section{Discussion}

Our study indicates that excessive EEG discontinuity assessed objectively with our automated software is associated with increased cerebral tissue injury on MRI, and is independently predictive of abnormal neurodevelopmental outcome in infants with HIE who have undergone $\mathrm{TH}$. This association was statistically significant at 24 and 48 hours of age, at $10 \mu \mathrm{V}$ and $15 \mu \mathrm{V}$ thresholds. Using a discontinuity cut-off of 30 s, PPVs of 75-90\% were obtained for MRIinferred outcomes and even higher PPVs for neurodevelopmental outcomes. The sensitivity for adverse neurodevelopmental outcome was greatest at 24 hours at $71 \%$ whereas the specificity at this time was at least $97 \%$. This highlights the importance of early EEG monitoring in identifying infants who are likely to benefit from therapeutic interventions.

Among infants with unfavourable MRI outcomes, the median discontinuity values dropped from 31.2 and 43.5 seconds at 24 hours to 2.7 and $20.3 \mathrm{~s}$ at 48 hours at $10 \mu \mathrm{V}$ and $15 \mu \mathrm{V}$ respectively, i.e. to below $30 \mathrm{~s}$ at both voltage thresholds. Hence in infants undergoing $\mathrm{TH}$, the PPV of a discontinuous trace for cerebral tissue injury on MRI is greater at 24 hours than at 48 hours with a substantial number of babies with poor MRI outcomes having EEG discontinuity values less than $30 \mathrm{~s}$ at 48 hours. This method may provide an important tool for risk stratification in early postnatal life when adjunctive therapies are most likely to be beneficial.

In earlier work we found that high seizure burden (seizures longer than 15 minutes per hour) was associated with brain tissue injury ${ }^{8}$, but we were unable to investigate the contribution of discontinuity, and its co-occurrence with seizure burden, to tissue injury and outcome due to technical limitations. The novel automated software we have developed for reliable quantification of discontinuity from cotside recordings of the EEG has now enabled such analysis: the evidence for collinearity between seizure burden and discontinuity was weak, which suggests that the point estimates of effect of each of these predictor variables in the model are likely to be reliable. Our novel automated software analysed single channel cross- 
cerebral EEG data, therefore it could be utilised with other aEEG/EEG software from which the raw EEG data can be extracted.

Previous studies have shown a relationship between features of multichannel EEG background $^{17}$ as well as limited channel EEG/aEEG measures ${ }^{18}$ and MRI outcomes in neonatal encephalopathy in the pre-cooling era, in that increased EEG depression is associated with more severe abnormality on cerebral MRI. Studies have shown the predictive value of EEG discontinuity lasting at least $30 \mathrm{~s}$ with adverse neurodevelopmental outcomes in infants with $\mathrm{HIE}^{6}$ as well as in groups of infants with broader neurologic diagnoses ${ }^{7}$ in the precooling era. Menache et al. ${ }^{7}$ reported that all 10 babies $(100 \%)$ with a predominant interburst interval duration of $30 \mathrm{~s}$ or greater had adverse neurologic outcomes; however, in that study the analogue EEG recordings may have been shorter with a minimum duration of 30 minutes. The use of automated quantification of background EEG activity has previously been demonstrated in infants ${ }^{1319}$. In a qualitative multichannel video-EEG study, Nash et al. ${ }^{9}$ found that a persistent burst suppression or continuous low voltage pattern beyond 24 hours of age in infants undergoing TH was highly predictive of brain injury on MRI. Using qualitative aEEG background patterns, a summary trend of the raw EEG, for term infants who had undergone TH, Thoresen et al. ${ }^{10}$ showed that the PPV of an abnormal aEEG background for poor outcome in cooled infants was $>90 \%$ at 24 hours in contrast to normothermic infants in whom the PPV of an abnormal trace reached $90 \%$ by age 12 hours. In the present study using an unbiased objective measure of discontinuity, we found similar PPVs at 24 and 48 hours of age in cooled babies and the PPV of the lower voltage threshold was greater.

Although our study was primarily designed to investigate the relationship between EEG discontinuity and MRI outcomes, we were also able to show an independent significant relationship between discontinuity and neurodevelopmental outcomes with a high PPV at 24 hours. We also found high concordance between MRI outcomes and later clinical outcomes at neurodevelopmental follow-up. The high early PPV of EEG discontinuity for abnormal MRI 
and neurodevelopmental outcomes may be a useful tool for early stratification of asphyxiated newborn babies for potentially beneficial adjunctive neuroprotective therapies.

\section{Acknowledgements}

We are grateful to Dr Courtney Wusthoff for her assistance with rating the seizure burden. We are grateful to all the nursing and medical staff from the study sites and all the families. 


\section{References}

1. Thornberg E, Thiringer K, Odeback A, et al. Birth asphyxia: incidence, clinical course and outcome in a swedish population. Acta Paediatrica 1995;84(8):927-32.

2. Levene ML, Kornberg J, Williams THC. The incidence and severity of post-asphyxial encephalopathy in full-term infants. Early Human Development 1985;11(1):21-26.

3. Garfinkle J, Sant'Anna GM, Wintermark P, et al. Cooling in the real world: Therapeutic hypothermia in hypoxic-ischemic encephalopathy. European Journal of Paediatric Neurology 2013;17(5):49297.

4. Edwards AD, Brocklehurst P, Gunn AJ, et al. Neurological outcomes at 18 months of age after moderate hypothermia for perinatal hypoxic ischaemic encephalopathy: synthesis and metaanalysis of trial data. British Medical Journal 2010;340.

5. Azzopardi D, Strohm B, Marlow N, et al. Effects of Hypothermia for Perinatal Asphyxia on Childhood Outcomes. New England Journal of Medicine 2014;371(2):140-49.

6. Wertheim D, Mercuri E, Faundez JC, et al. Prognostic value of continuous electroencephalographic recording in full-term infants with hypoxic-ischemic encephalopathy. Archives of Disease in Childhood 1994;71(2):F97-F102.

7. Menache CC, Bourgeois BFD, Volpe JJ. Prognostic value of neonatal discontinuous EEG. Pediatric Neurology 2002;27(2):93-101.

8. Shah DK, Wusthoff CJ, Clarke $P$, et al. Electrographic seizures are associated with brain injury in newborns undergoing therapeutic hypothermia. Archives of Disease in Childhood-Fetal and Neonatal Edition 2014;99(3):F219-F24.

9. Nash KB, Bonifacio SL, Glass HC, et al. Video-EEG monitoring in newborns with hypoxic-ischemic encephalopathy treated with hypothermia. Neurology 2011;76(6):556-62.

10. Thoresen M, Hellstrom-Westas L, Liu X, et al. Effect of Hypothermia on Amplitude-Integrated Electroencephalogram in Infants With Asphyxia. Pediatrics 2010;126(1):E131-E39.

11. Azzopardi DV, Strohm B, Edwards AD, et al. Moderate hypothermia to treat perinatal asphyxial encephalopathy. The New England journal of medicine 2009;361(14):1349-58.

12. UK TOBY Cooling Register Clinician's Handbook https://www.npeu.ox.ac.uk/files/downloads/tobyregister/Register-Clinicans-Handbook1-v4-0706-10.pdf.

13. Wertheim DFP, Eaton DGM, Oozeer RC, et al. A new system for cotside display and analysis of the preterm neonatal electroencephalogram. Developmental Medicine and Child Neurology 1991;33(12):1080-86.

14. Werner S, Bickford R, Stockard-Pope J. Atlas of Neonatal Electroencephalography. New York: Raven Press, 1977.

15. Andre M, Lamblin MD, d'Allest AM, et al. Electroencephalography in premature and full-term infants. Developmental features and glossary. Neurophysiologie Clinique-Clinical Neurophysiology 2010;40(2):59-124.

16. Rutherford MA, Pennock JM, Counsell SJ, et al. Abnormal magnetic resonance signal in the internal capsule predicts poor neurodevelopmental outcome in infants with hypoxic-ischemic encephalopathy. Pediatrics 1998;102(2):323-28.

17. Biagioni $\mathrm{E}$, Mercuri $\mathrm{E}$, Rutherford $\mathrm{M}$, et al. Combined use of electroencephalogram and magnetic resonance imaging in full-term neonates with acute encephalopathy. Pediatrics 2001;107(3):461-68.

18. Shah DK, Lavery S, Doyle LW, et al. Use of 2-channel bedside electroencephalogram monitoring in term-born encephalopathic infants related to cerebral injury defined by magnetic resonance imaging. Pediatrics 2006;118(1):47-55.

19. Lofhede J, Thordstein M, Lofgren N, et al. Automatic classification of background EEG activity in healthy and sick neonates. Journal of Neural Engineering 2010;7(1). 
Table 1: Characteristics of study infants

\section{Perinatal Characteristics (total neonates $=49$ )}

Male sex

Median birth weight, grams (range)

Median Apgar score at 10 min (IQR), n=44

Median first $\mathrm{pH}$, by cord or within $1 \mathrm{~h}$, $\mathrm{mmol} / \mathrm{L}$ (IQR), $\mathrm{n}=48$

Median first base deficit, by cord or within 1h, mmol/L (IQR), n=46

Need for respiratory support at $10 \mathrm{~min}$ age

Median age when heart rate first $>100 / \mathrm{min}$, minutes (IQR), $\mathrm{n}=48$

Median age at first gasp, minutes (range) $\mathrm{n}=41$

Antenatal sentinel event identified

Suspected clinical seizures

Treated with anticonvulsants

Received sedation during cooling

Median age at start of aEEG monitoring (range), $\mathrm{n}=47$

Median duration of aEEG monitoring (range), $\mathrm{n}=48$

Any seizures identified on aEEG/EEG

High seizure burden on aEEG/EEG

Epoch analysed at 24 and 48 hours, minutes (range)

Median age at MRI, days (interquartile range)

Severe pattern of injury on MRI (group 1)

Unfavourable neurodevelopmental outcome
$29(59 \%)$

$18(37 \%)$

$32(65 \%)$

3360 (2450-4560)

$6(4,8)$

$6.91(6.81,7.10)$

$-16.9(-11.3,-21.5)$

$37(76 \%)$

$2(1,5)$

$5(0,20)$

$8(16 \%)$

$36(73 \%)$

$37(76 \%)$

$42 / 46(91 \%)$

4h $50 \mathrm{~m}$ (15m to $11 \mathrm{~h} 20 \mathrm{~m})$

$89 \mathrm{~h} 53 \mathrm{~m}$ (13h $20 \mathrm{~m}$ to $168 \mathrm{~h} 58 \mathrm{~m})$

$119(80,120)$

$11(8,14)$

$17 / 49(35 \%)$

$14 / 43(33 \%)$ 
Table 2: Discontinuity values in seconds, MRI, seizure burden and neurodevelopment for the study babies.

\begin{tabular}{|c|c|c|c|c|c|c|c|}
\hline Baby & $\begin{array}{l}\mathrm{DC}^{\dagger} \\
10 \mu \mathrm{V} \text { at } \\
24 \mathrm{~h}\end{array}$ & $\begin{array}{l}\mathrm{DC}^{\dagger} \\
15 \mu \mathrm{V} \text { at } \\
24 \mathrm{~h}\end{array}$ & BGT & PLIC & $\begin{array}{l}\text { White } \\
\text { Matter }\end{array}$ & $\begin{array}{l}\text { Seizure } \\
\text { Burden }\end{array}$ & $\begin{array}{l}\text { Neurodevelopmental } \\
\text { Outcome }\end{array}$ \\
\hline 1 & 0 & 0 & Mild & Normal & Mild & None & Normal \\
\hline 2 & 0 & 0 & Mild & Normal & Moderate & None & Normal \\
\hline 3 & 0 & 0 & Mild & Normal & Moderate & None & Normal \\
\hline 4 & 0 & 0 & Mild & Normal & Normal & None & Normal \\
\hline 5 & 0 & 0 & Mild & Normal & Mild & None & Normal \\
\hline 6 & 0 & 0 & Mild & Normal & Normal & Low & Normal \\
\hline 7 & 0 & 0 & Normal & Normal & Mild & Low & Normal \\
\hline 8 & 0 & 0 & Mild & Normal & Mild & None & Normal \\
\hline 9 & 0 & 0 & Mild & Normal & Moderate & None & Bilateral SN hearing loss \\
\hline 10 & 0 & 0 & Normal & Normal & Normal & None & $\mathrm{n} / \mathrm{a}$ \\
\hline 11 & 0 & 0 & Normal & Normal & Normal & None & $\mathrm{n} / \mathrm{a}$ \\
\hline 12 & 0 & 0.1 & Normal & Normal & Normal & None & Normal \\
\hline 13 & 0 & 0.2 & Mild & Normal & Normal & None & Normal \\
\hline 14 & 0 & 0.2 & Normal & Normal & Severe & High & Normal \\
\hline 15 & 0 & 0.2 & Mild & Normal & Moderate & High & Normal \\
\hline 16 & 0 & 0.2 & Mild & Normal & Moderate & Low & $\begin{array}{c}\text { Seizures and unilateral } \\
\text { hearing loss }\end{array}$ \\
\hline 17 & 0 & 1.2 & Normal & Normal & Mild & None & Normal \\
\hline 18 & 0 & 3.3 & Moderate & Partially Impaired & Severe & High & Normal \\
\hline 19 & 0 & 21.5 & Mild & Normal & Moderate & High & Normal \\
\hline 20 & 0.1 & 0.8 & Moderate & Normal & Severe & High & Mild speech delay \\
\hline 21 & 0.1 & 1.8 & Mild & Normal & Normal & None & Normal \\
\hline 22 & 0.1 & 12.5 & Normal & Normal & Normal & None & Normal \\
\hline 23 & 0.2 & 1.7 & Mild & Normal & Mild & None & $\mathrm{n} / \mathrm{a}$ \\
\hline 24 & 0.2 & 3.3 & Mild & Normal & Mild & High & Normal \\
\hline 25 & 0.3 & 18.6 & Normal & Partially Impaired & Normal & None & Normal \\
\hline 26 & 0.6 & 3.5 & Moderate & Normal & Mild & None & Normal \\
\hline 27 & 0.6 & 9.9 & Normal & Normal & Mild & Low & Normal \\
\hline 28 & 0.9 & 11.1 & Severe & Severely Impaired & Severe & High & Dyskinetic CP \\
\hline 29 & 1.2 & 8.6 & Normal & Normal & Severe & High & Normal \\
\hline 30 & 2.4 & 16 & Moderate & Normal & Moderate & None & $\mathrm{n} / \mathrm{a}$ \\
\hline 31 & 2.4 & 18.5 & Normal & Normal & Normal & Low & $\begin{array}{l}\text { Bilateral SN hearing loss, } \\
\text { significant receptive and } \\
\text { expressive speech delay }\end{array}$ \\
\hline 32 & 2.9 & 9.4 & Mild & Normal & Moderate & None & Normal \\
\hline 33 & 3.6 & 9.3 & Mild & Normal & Mild & High & Normal \\
\hline 34 & 4.8 & 24.3 & Normal & Normal & Normal & Low & $\mathrm{n} / \mathrm{a}$ \\
\hline 35 & 5 & 19.6 & Mild & Normal & Mild & High & Mild delay \\
\hline 36 & 7.4 & 23.3 & Mild & Normal & Mild & Low & Normal \\
\hline 37 & 7.9 & 18.8 & Moderate & Severely Impaired & Mild & None & Normal \\
\hline 38 & 25.2 & 47.3 & Normal & Normal & Mild & Low & $\mathrm{n} / \mathrm{a}$ \\
\hline 39 & 27.7 & 50.4 & Mild & Normal & Mild & High & Normal \\
\hline 40 & 31.2 & 43.5 & Severe & Severely Impaired & Moderate & Low & Quadriplegic CP \\
\hline 41 & 47.3 & 50.3 & Moderate & Severely Impaired & Mild & High & $\begin{array}{l}\text { Spastic quadriplegic CP, } \\
\text { GDD, SN hearing loss and } \\
\text { dystonia }\end{array}$ \\
\hline 42 & 49.2 & 54.4 & Severe & Severely Impaired & Moderate & High & $\begin{array}{l}\text { GDD, microcephaly, visual } \\
\text { impairment, dystonia with } \\
\text { epilepsy and GORD }\end{array}$ \\
\hline 43 & 51.4 & 56.5 & Severe & Severely Impaired & Moderate & High & Quadriplegic CP \\
\hline 44 & 51.8 & 56 & Severe & Severely Impaired & Severe & Low & Died \\
\hline 45 & 54.5 & 58 & Moderate & Severely Impaired & Severe & High & $\begin{array}{l}\text { Global speech delay, autism } \\
\text { and seizures }\end{array}$ \\
\hline 46 & 55.3 & 57.9 & Moderate & Severely Impaired & Moderate & High & Died \\
\hline 47 & 58.6 & 59.9 & Severe & Severely Impaired & Moderate & Low & Died \\
\hline 48 & 58.9 & 59.7 & Mild & Partially Impaired & Normal & High & Died \\
\hline 49 & 59.4 & 59.5 & Severe & Severely Impaired & Moderate & High & Spastic quadriplegic CP \\
\hline
\end{tabular}


${ }^{\dagger}$ DC: Discontinuity (at stated threshold and time epoch).

${ }^{\star}$ Low seizure burden includes either no or sporadic seizures, high seizure burden includes either frequent seizures or status epilepticus.

BGT: Basal ganglia and thalami, PLIC: Posterior limb of the internal capsule, CP: Cerebral palsy, GDD: Global developmental delay, GORD: Gastro-oesophageal reflux disease. SN, sensori-neural. 
Table 3: Univariable analyses for the association between risk factors and poor outcome on MRI (first grid) and multivariable regression model assessing significant factors in univariable analyses against poor MRI outcome (second grid). Predictive values for unfavourable outcome with a mean discontinuity $>30$ s on MRI (third grid) and neurodevelopmental outcome (fourth grid).

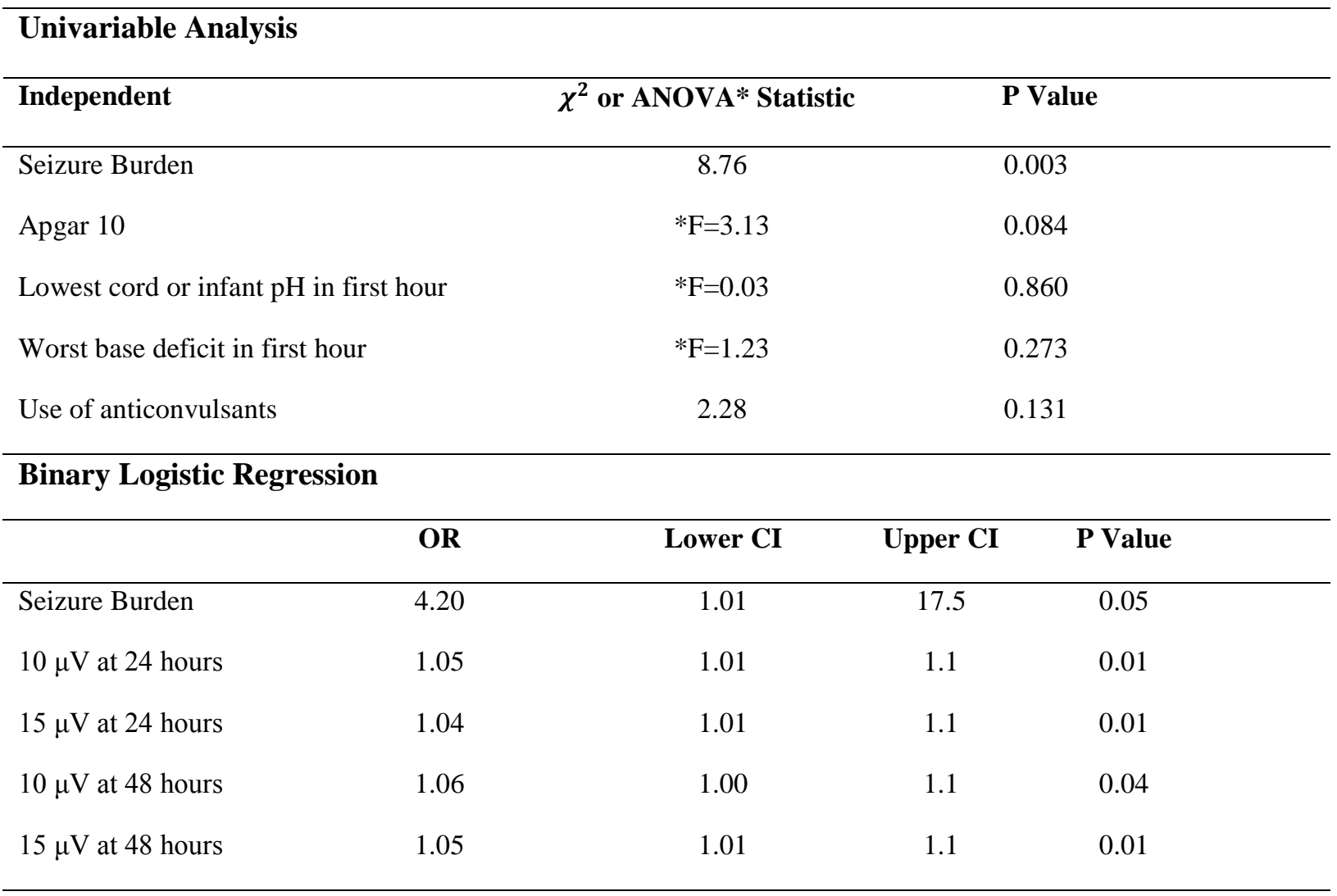

Predictive Values of Discontinuity (>30s) for Unfavourable MRI Outcomes

\begin{tabular}{|c|c|c|c|c|c|c|}
\hline & Sensitivity & Specificity & PPV & NPV & $\begin{array}{l}\text { Area under } \\
\text { ROC }\end{array}$ & $P$ Value \\
\hline $10 \mu \mathrm{V}$ at 24 hours & $53 \%$ & $94 \%$ & $82 \%$ & $79 \%$ & 0.80 & 0.001 \\
\hline $15 \mu \mathrm{V}$ at 24 hours & $53 \%$ & $91 \%$ & $75 \%$ & $78 \%$ & 0.79 & 0.001 \\
\hline $10 \mu \mathrm{V}$ at 48 hours & $41 \%$ & $97 \%$ & $87 \%$ & $76 \%$ & 0.78 & $<0.001$ \\
\hline $15 \mu \mathrm{V}$ at 48 hours & $47 \%$ & $94 \%$ & $80 \%$ & $77 \%$ & 0.81 & 0.001 \\
\hline \multicolumn{7}{|c|}{ Predictive Values of Discontinuity (>30s) for Unfavourable Neurodevelopmental Outcomes } \\
\hline & Sensitivity & Specificity & PPV & NPV & OR & $95 \% \mathrm{CI}$ \\
\hline $10 \mu \mathrm{V}$ at 24 hours & $71 \%$ & $100 \%$ & $100 \%$ & $88 \%$ & - & - \\
\hline $15 \mu \mathrm{V}$ at 24 hours & $71 \%$ & $97 \%$ & $91 \%$ & $88 \%$ & 9.6 & $1.48,62.66$ \\
\hline $10 \mu \mathrm{V}$ at 48 hours & $50 \%$ & $97 \%$ & $87 \%$ & $80 \%$ & 6.4 & $1.02,40.33$ \\
\hline $15 \mu \mathrm{V}$ at 48 hours & $64 \%$ & $96 \%$ & $90 \%$ & $85 \%$ & 8.5 & $1.31,54.78$ \\
\hline
\end{tabular}

OR: Odds ratio, CI: Confidence interval, PPV: Positive predictive value, NPV: Negative predictive value, ROC: Receiver operating characteristic. 
Figure 1: Plots comparing mean discontinuity and MRI outcome (favourable or unfavourable). The left panels shows mean discontinuity at 24 hours and the right panels at 48 hours. The top panels utilise a $10 \mu \mathrm{V}$ threshold and the bottom panels a $15 \mu \mathrm{V}$ threshold. The box edges show the interquartile range and the whiskers the range. 
Figure 2: Plots comparing mean discontinuity and neurodevelopmental outcome. The left panels shows mean discontinuity at 24 hours and the right panels at 48 hours. The top panels utilise a $10 \mu \mathrm{V}$ threshold and the bottom panels a $15 \mu \mathrm{V}$ threshold. The box edges show the interquartile range and the whiskers the range. 


\section{Copyright Statement:}

The Corresponding Author has the right to grant on behalf of all authors and does grant on behalf of all authors, an exclusive licence (or non-exclusive for UK Crown Employees) on a worldwide basis to the BMJ Publishing Group Ltd, and its Licensees to permit this article (if accepted) to be published in Archives of Disease in Childhood and any other BMJPGL products and to exploit all subsidiary rights, as set out in our licence. 